\title{
Voltammetric Study of the Kolbe Reaction at a Rotating Platinum Anode
}

\author{
M.J. Allen, W.G. Pierson and A.V. Celiano \\ Research Division, Melpar, Inc., Falls Church, Virginia and Department of Chemistry, \\ Seton Hall University, South Orange, New Jersey, U.S.A.
}

\begin{abstract}
A number of aliphatic carboxylic acids were investigated voltammetrically in anhydrous methanol at a rotating platinum anode $v s$. a silver-silver chloride reference electrode utilizing a mercury-mercurous acetate cathode. It was found that those acids which yield Kolber dimers require a relatively lower potential for the formation of the carboxylate radical than those acids which do not dimerize. Of interest too are the findings which indicate that acids which do give a Kolbe dimer demonstrate in most instances the requirement of a critical current density and concentration. As far as can be determined there is apparently no correlation between structure and half-wave potentials in those acids which yield dimers. As these potentials represent in all probability the formation of the free-radical the lack of correlation is not unexpected in view of similar findings related to pinacol formation. Based on the experimental findings it appears that the first step in the reaction i.e., the formation of the carboxylate radical, is potential dependent and that the subsequent dimerization is only dependent on the resultant free-radical concentration at the electrode surface.
\end{abstract}

The objective of this investigation was to study the voltammetric behavior of potassium acetate and other aliphatic carboxylic acids in an anhydrous methanol medium at a smooth platinum anode in an attempt to gain more information about the processes occurring in the Kolbe reaction.

Three theories have been presented concerning the mechanism of the Kolbe reaction: the discharged ion theory by Brown and Walker ${ }^{1)}$, the acetyl peroxide theory by Fichter ${ }^{2}$, and the hydrogen peroxide theory by Glasstone and Hickling ${ }^{3)}$.

Salauze $^{4}$ ) reported in 1925 that the Kolbe reaction went in a methanol medium as well as or better than the aqueous medium used by Kolbe ${ }^{5}$. Conditions such as electrode material, current density, temperature and concentration of depolarizer were reportedly not nearly so critical with the methanol medium. This work, in addition to the experiments by Glasstone and Hickling ${ }^{6}$ in which added quantities of hydrogen peroxide to solutions of acetates produced negligible amounts of Kolbe dimer, has cast serious doubts on the accuracy of the hydrogen peroxide theory.

In 1956, Wilson and Lippincott ${ }^{7}$ conducted a series of kinetic studies, the results of which have all but eliminated the hydrogen peroxide and acetyl peroxide theories from serious consideration.

The most commonly accepted theory at the present time is the modernized version of the discharged ion theory of Brown and Walker in which the rate determining step is the lose of one electron to the anode by the acetate ion to yield an acetate radical which spontaneously splits off carbon dioxide. The methyl radical formed is then free to react with a second methyl radical to give the Kolbe dimer, ethane.

If we accept this theory as correct, the formation of the carboxylate radical should give an irreversible voltammetric step in which the diffusion current varies linearly with concentration.

\section{Experimental}

An anhydrous methanol medium was chosen for these experiments because it electrolyzes at a higher potential than water, and was less likely to interfere with the voltammetric step of the anion. Also, the lower sensitivity of the Kolbe reaction to electrode material, temperature, current density and concentration of depolarizer in a methanol medium allows a greater variation of conditions without interfering with the mechanism of the reaction. This was desirable in the study, as the effect of various concentra- 
tions of anion was to be investigated.

The use of anhydrous methanol for the solvent did, however, complicate the problem to some extent in that a suitable reference electrode had to be found for use with this medium. This problem was solved by use of a silver-silver chloride reference electrode prepared according to the method described by Brown ${ }^{8)}$. The electrode was found completely reliable under all conditions of these experiments. Recently Gillet ${ }^{9}$ utilized the same type electrode for his studies in anhydrous ether.

Although the silver-silver chloride made an excellent reference electrode, its design made it entirely unsuitable for use as an unpolarizable cathode. A large platinum disc and a mercury pool were both tried and found lacking because they could not maintain a consta potential throughout the entire applied voltage range used in these experiments. The solution to this problem was the use of a mercurymercurous acetate cathode. Although the potential was found to vary slightly from one carboxylate ion concentration to the next, it remained entirely unchanged throughout the entire applied voltage range at any given concentration of carboxylate. Because of this slight variation of potential with carboxylate ion concentration, mercury-mercurous acetate could not be used as a reference electrode. Its unchangeable character at any single concentration, however, made it an ideal unpolarizable cathode.

A final problem which had to be overcome was the high resistance of a methanolic solution. Correction for IR drop is a simple matter, but with the cell in its final form, the applied potential needed to discharge the carboxylate ion and to overcome the IR drop was too high to use one of the commercially available polarographs. In some cases, an applied potential of over 4 volts was requird.

The Redoxotrol Mark IV described by Allen $^{10)}$ was easily adapted to this use, and was found to be quite adequate. This instrument is capable of any output up to 100 volts, and is equipped with a motordriven variable voltage transformer which gives an almost linear voltage-time curve.

The current-voltage curves were plotted with a Varian Gll 20 milli-volt recorder. When this instrument is used with the Redoxotrol at its most sensitive range, it gives a full scale reading of $4 \mathrm{~mA}$. During these experiments, the Redoxotrol was set to change output potential at a rate of $1.4 \mathrm{~V}$ per minute, and the recorder set to run at a speed of 2 inches per minute.

The electrode used in these experiments is shown in Fig. 1. The cell used is described in Fig. 2.

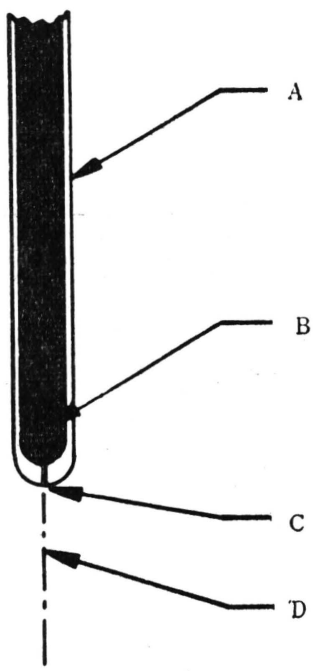

A : Mercury filled glass tube

B : Platinum wire sealed in glass

C : Exposed platinum disk

$\mathrm{D}:$ Axis of rotation

Fig. 1 Rotating platinum disk microelectrode

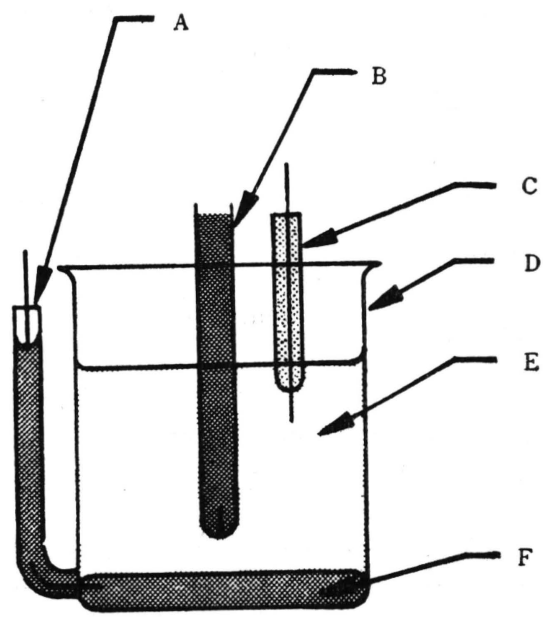

A : Mercury filled cathode contact

B : Rotating platinum disk microanode

C : Silver-silver chloride

$\mathrm{D}: 100 \mathrm{~m} l$ beaker cell E : Test solution

F : Mercury mercurous acetate unpolarizable cathode

Fig. Voltammetry cell 
The anode consisted of a length of platinum wire, 0.02 inch in diameter, sealed in the end of a soft glass tube and then cut at the glass line to expose only a disc of platinum with an area of 0.2025 $\mathrm{mm}^{2}$. The disc was buffed to a high polish with fine paper and cleaned with boiling nitric acid. The speed of anode rotation was a constant $600 \mathrm{rpm}$.

The cathode was prepared by sprinkling a small amount of mercurous acetate on a pool of clean mercury and then swirling the mercury until a thin skin of mercurous acetate completely covered the mercury surface. The area of the cathode was approximately $1735 \mathrm{~mm}^{2}$, or 8500 times larger than the anode. The test solution was added to the cell in such a way as to not disturb the surface of the cathode, and the anode was placed $5 \mathrm{~mm}$ above the cathode. If the anode were placed any closer, its rotation. would have disturbed the cathode surface.

The reference electrode was placed in the solution close to the anode, but out of the lines of electrical force.

All measurements were made at a controlled temperature of $25^{\circ}$. The cell resistance was measured between the anode and the cathode with a conductivity bridge (Leeds and Northrup No. 4866).

The current-voltage curve was obtained in the following way: First, a current-time curve was plotted in the usual manner. Then a plot was made of anode voltage $v s$. reference voltage, and the values so obtained were transposed onto the current-time curve. Examination of the voltage-time curve (Fig. 3) will show that the change of voltage with time is linear at all but the very low values, and this curve had reached the point of linearity long before the voltammetric step had been reached.

The unpolarizability of the cathode was checked by plotting the cathodé potential against reference potential and in every case a straight line parallel to the base line and very close to it was ob-

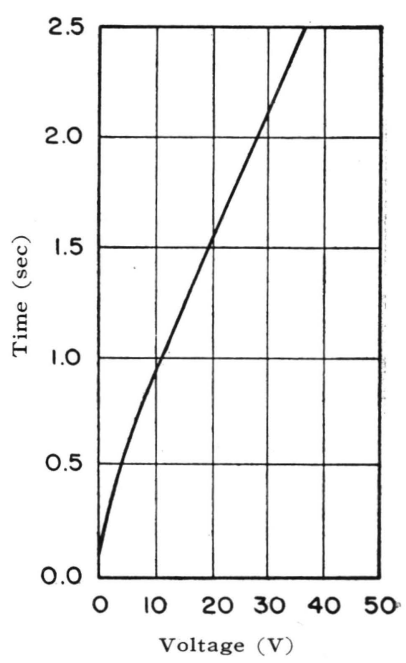

Fig. 3 Voltage vs. time curve obtained from redoxotrol served.

All of the voltages given in this paper are with respect to the silver-silver chloride reference electrode, and no effort has been made to convert these values to the more common N.H.E. or S.C.E. scales. Parsons $^{12)}$ gives the standard potential of the silver-silver chloride electrode in $100 \%$ methanol to be -0.013 volts on the hydrogen electrode scale. No attempt has been made to determine whether the presence of acetate or other carboxylate ion affects this value.

Furthermore as we are dealing with solutions of extremely high resistance it is perhaps more reasonable to look upon the diffusion currents and half-wave potentials as "apparent" diffusion currents and half-wave potentials.

Table 1 Sodium Acetate

\begin{tabular}{l|c|c|c}
\hline \hline Conc. & Diffusion current & Cell resistance & $\begin{array}{c}\text { Corrected half-wave } \\
\text { potential }\end{array}$ \\
\hline $0.05 M$ & $0.32 \mathrm{~mA}$ & 2900 ohms & +1.64 volts \\
0.1 & 0.36 & 2500 & 1.65 \\
0.2 & 0.66 & 1663 & 1.65 \\
0.4 & 1.36 & 1186 & 1.57 \\
0.6 & 1.96 & 984 & 1.46 \\
0.8 & 2.70 & 922 & 1.36 \\
1.0 & 3.28 & 888 & 1.25 \\
\hline
\end{tabular}

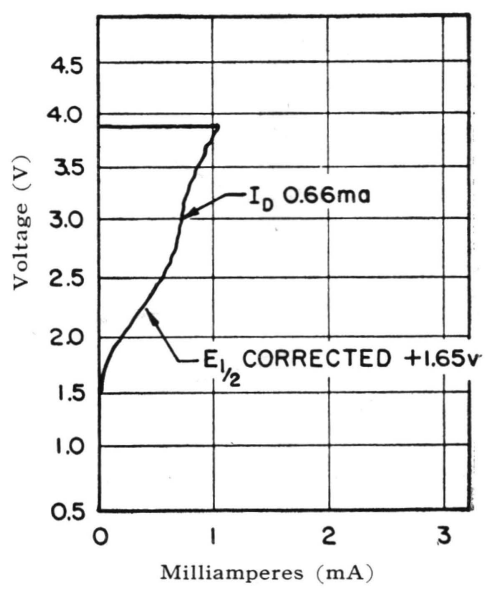

Fig. $4 \quad 0.2 M$ potassium acetate 
A typical acetate current-voltage curve is shown in Fig. 4. The results for the various concentration investigated are shown in Table 1.

A plot of diffusion current vs. concentration shows a linear increase in $i_{d}$ with concentration.

With the background experience gained using potassium acetate, we turned our attention to other organic acids known to give Kolbe dimers. Fig. 5 shows the voltammetric step with sodium valerate when the electrolyte is dilute, and any product formed is quickly dissolved in the medium and diffuses away from the anode. As a result, the methanol is able to react, and this is seen as a rise in the curve after the diffusion current has been passed or another step has begun.

Fig. 6 shows a typical current-voltage curve where the product formed is sufficient to suppress any methanol reaction, and the last step does not appear. The result is a voltammetric curve in which the solvent does not react, and the diffusion current is represented by a flat line.

Fig. 7 illustrates a curve in which the product may be formed too rapidly to diffuse away from the electrode, and as a result, the valerate ions must diffuse through a different medium with a different diffusion coefficient. The peak is probably a result of the ions diffusing through methanol, and the drop occurs when they begin to diffuse possibly through octane. When this type of peak is present, in all probability we are not dealing with a $100 \%$ methanol medium, but rather a hydrocarbon medium. This layer of hydrocarbon also produces an additional IR drop, and the half-wave potentials should include this factor, in order to be completely accurate. Unfortunately, it is quite difficult to measure this additional resistance.

Tabulated results obtained with sodium valerate are given in Table 2 .

Table 2 Sodium Valerate

\begin{tabular}{l|c|c|c}
\hline \hline Conc. & Diffusion current & Cell resistance & $\begin{array}{c}\text { Corrected half-wave } \\
\text { potential }\end{array}$ \\
\hline $0.05 M$ & $0.14 \mathrm{~mA}$ & $4820 \mathrm{ohms}$ & +1.47 volts \\
0.1 & 0.25 & 3100 & 1.52 \\
0.2 & 0.46 & 2160 & 1.97 \\
0.3 & 0.54 & 1850 & 1.75 \\
0.4 & 0.57 & 1650 & 1.63 \\
0.5 & 0.60 & 1595 & 1.73 \\
\hline
\end{tabular}

From the plot of diffusion current vs. concentration it appears that a critical concentration $(2.025 M)$ is reached where the rate of dissolution of product begins to interfere with the rate of diffusion of the valerate. Since current is a measure of the reaction rate, and current density a measure of free radical concentraton in the vicinity of the anode, a critical current density

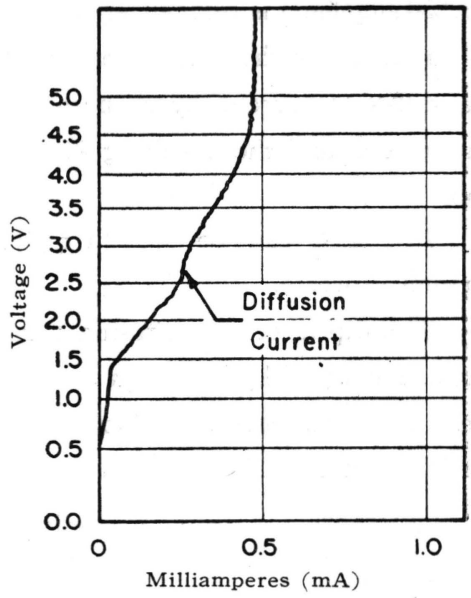

Fig. $50.1 M$ sodium valerate $\left(\mathrm{CH}_{3} \mathrm{CH}_{2} \mathrm{CH}_{2} \mathrm{CH}_{2} \mathrm{COONa}\right)$

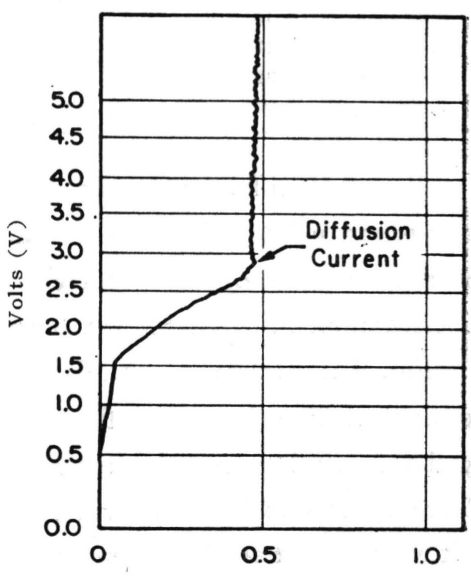

Milliamperes (mA)

Fig. $6 \quad 0.2 M$ sodium valerate $\left(\mathrm{CH}_{3} \mathrm{CH}_{2} \mathrm{CH}_{2} \mathrm{CH}_{2} \mathrm{COONa}\right)$

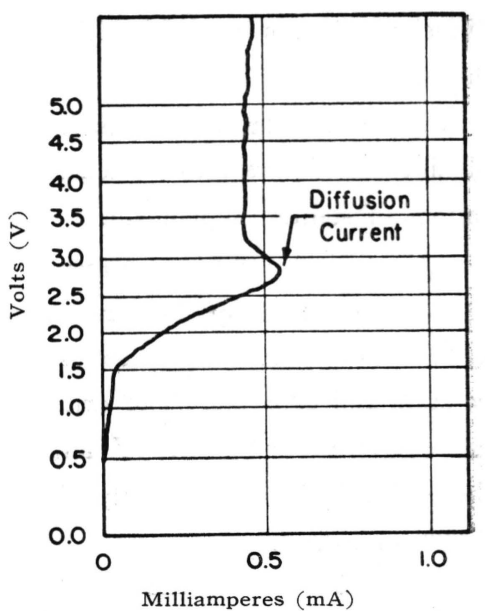

Fig. $7 \quad 0.3 M$ sodium valerate $\left(\mathrm{CH}_{3} \mathrm{CH}_{2} \mathrm{CH}_{2} \mathrm{CH}_{2} \mathrm{COONa}\right)$ 
$\left(0.256 \mathrm{amp} / \mathrm{cm}^{2}\right)$ is reached at which the free radical concentration is optimum for the Kolbe reaction to occur. This, then, changes the diffusion current by changing the nature of the medium at the anode, and the resulting diffusion coefficient of the valerate ion.

In Fig. 8 can be seen a typical current-voltage curve for sodium caproate.

The dip after the diffusion current is probably caused by the accumulation of hydrocarbon around the anode. This dip is not present in the more dilute solution. The results obtained with various concentrations are shown in Table 3 .

Table 3 Sodium Caproate

\begin{tabular}{l|l|l|c}
\hline \hline Conc. & Diffusion current & Cell resistance & $\begin{array}{c}\text { Corrected half-wave } \\
\text { potential }\end{array}$ \\
\hline $0.05 M$ & $0.175 \mathrm{~mA}$ & $4080 \mathrm{ohms}$ & +1.70 volt \\
0.1 & 0.22 & 2662 & 1.81 \\
0.2 & 0.31 & 1812 & 1.92 \\
0.3 & 0.34 & 1503 & 1.95 \\
0.4 & 0.345 & 1365 & 1.92 \\
0.5 & 0.355 & 1270 & 1.88 \\
\hline
\end{tabular}

A plot of diffussion current $v s$. concentration shows again an intersecting point which may indicate, as with the case of sodium valerate, that the optimum current density is $0.165 \mathrm{amp} / \mathrm{cm}^{2}$ and concentration, $2.023 M$ for the formation of Kolbe dimer.

Fig. 9 illustrates a typical voltammetric curve with sodium heptanoate. The drop after the diffusion point is probably caused by the accumulation of hydrocarbon at this low concentration. There appears to be an intermediate step in the vicinity of the half-wave potential. This step occurs only with the larger anions and is probably caused by a small amount of excess methoxide anion used in the preparation of the salt. It only shows up with the larger acids because of their low diffusion currents. Larger

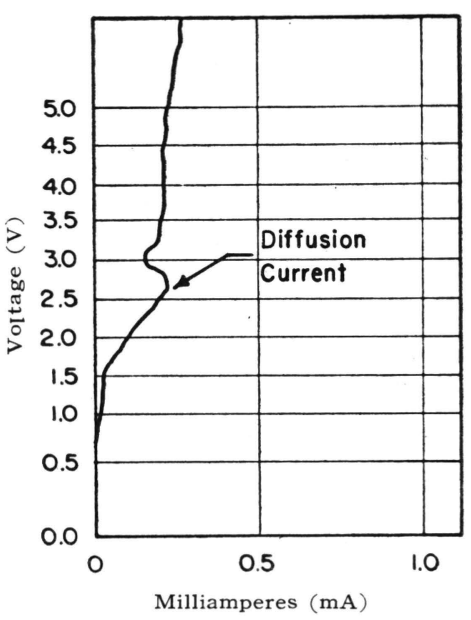

Fig. $8 \quad 0.1 M$ sodium caproate $\left(\mathrm{CH}_{3} \mathrm{CH}_{2} \mathrm{CH}_{2} \mathrm{CH}_{2} \mathrm{COONa}\right)$

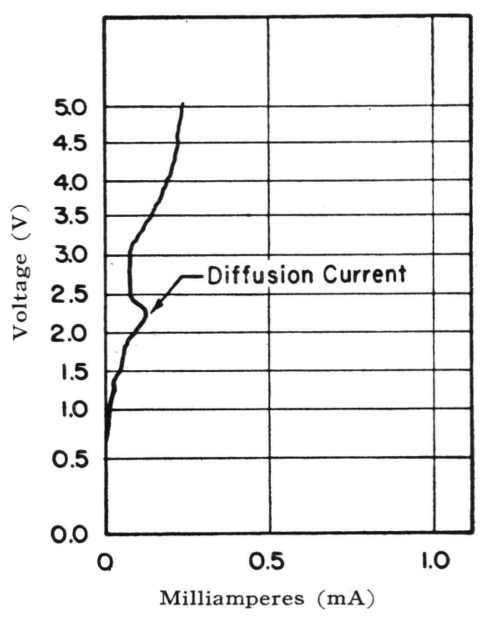

Fig. $90.1 \mathrm{M}$ sodium hepatanoate $\left[\mathrm{CH}_{3}\left(\mathrm{CH}_{2}\right)_{5} \mathrm{COONa}\right]$ diffusion currents probably hide this small step.

Table 4 relates the results obtained with various concentrations of sodium heptanoate.

A plot of diffusion current vs. concentration of heptanoate again follows the pattern set by valerate and caproate. The critical concentration is $0.3 M$ and the critical current density is $0.083 \mathrm{amps} / \mathrm{cm}^{2}$.

Table 5 describes the results obtained with sodium iso-valerate.

As can be seen in Fig. 10,
Table 4 Sodium Heptanoate

\begin{tabular}{l|l|l|c}
\hline \hline Conc. & Diffusion current & Cell resistance & $\begin{array}{c}\text { Corrected half-wave } \\
\text { potential }\end{array}$ \\
\hline $0.05 M$ & $0.12 \mathrm{~mA}$ & $5000 \mathrm{ohms}$ & +1.50 volts \\
0.1 & 0.13 & 3350 & 1.64 \\
0.2 & 0.15 & 2295 & 1.73 \\
0.3 & 0.17 & 1942 & 1.64 \\
0.4 & 0.17 & 1752 & 1.66 \\
0.5 & 0.17 & 1700 & 1.66 \\
\hline
\end{tabular}

Table 5 Sodium Iso-valerate

\begin{tabular}{l|c|c|c}
\hline \hline Conc. & Diffusion current & Cell resistance & $\begin{array}{c}\text { Corrected half-wave } \\
\text { potential }\end{array}$ \\
\hline $0.05 M$ & $0.17 \mathrm{~mA}$ & 4900 ohms & +1.39 volts \\
0.1 & 0.33 & 3090 & 1.50 \\
0.2 & 0.65 & 2100 & 1.57 \\
0.3 & 0.90 & 1754 & 1.62 \\
0.4 & 1.15 & 1575 & 1.40 \\
0.5 & 1.40 & 1342 & 1.62 \\
\hline
\end{tabular}


the unusual aspect of the iso-valerate curves is the lack of depression in the curve at all concentrations investigated after the diffusion current has been reached. Also, a plot of diffusion current vs. concentration shows only a slight break in the vicinity of $0.2 \mathrm{M}$ concentration.

A possible explanation for this departure from most other curves obtained is that the yield of Kolbe dimer is relatively lower, and the product formed only slightly soluble in methanol. As a result, the diffusion layer is essentially

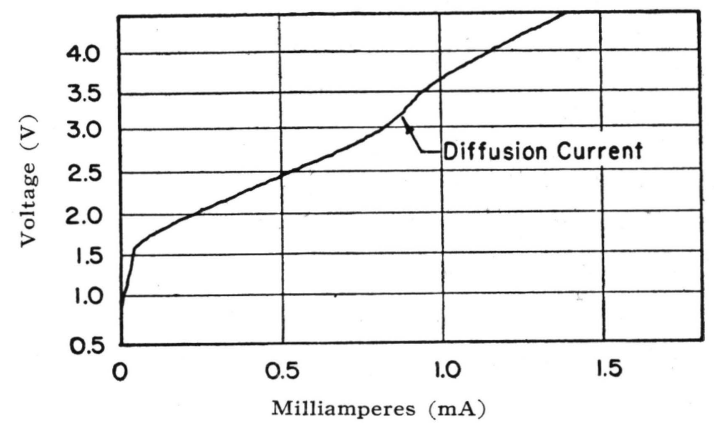

Fig. $10 \quad 0.3 M$ sodium iso-valerate $\left[\left(\mathrm{CH}_{3}\right)_{2} \mathrm{CHCH}_{2} \mathrm{COONa}\right]$ composed of methanol uncontaminated by hydrocarbon. This curve is similar to those with acetate, in which a soluble product is obtained, and acetylglycinate where no Kolbe product is obtained.

A typical curve obtained with sodium iso-caproate (Fig. 11) shows a return to the pattern obtained with sodium valerate.

Table 6 tabulates the results obtained with various concentrations of iso-caproate:

Table 6 Sodium Iso-caproate

\begin{tabular}{l|c|c|c}
\hline \hline Conc. & Diffusion current & Cell resistance & $\begin{array}{c}\text { Corrected half-wave } \\
\text { potential }\end{array}$ \\
\hline $0.05 M$ & $0.16 \mathrm{~mA}$ & 4000 ohms & +1.83 volts \\
0.10 & 0.26 & 2580 & 1.86 \\
0.14 & 0.33 & 2130 & 1.80 \\
0.20 & 0.36 & 1768 & 1.94 \\
0.30 & 0.39 & 1480 & 1.92 \\
0.40 & 0.41 & 1325 & 1.98 \\
0.50 & 0.44 & 1245 & 1.98 \\
\hline
\end{tabular}

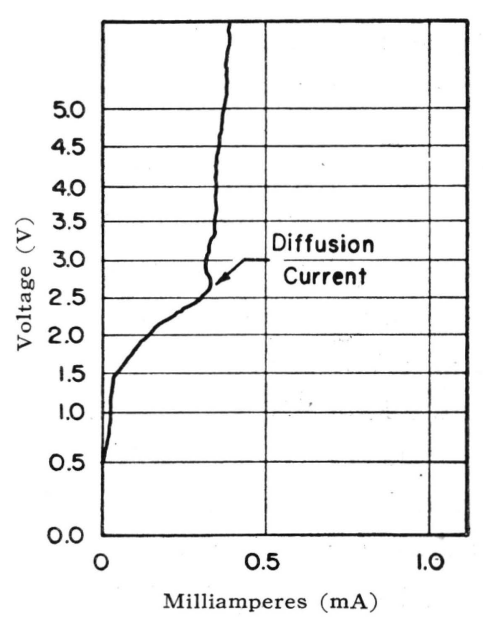

Fig. $110.14 \mathrm{M}$ sodium iso-caproate $\left[\left(\mathrm{CH}_{3}\right)_{2} \mathrm{CHCH}_{2} \mathrm{CH}_{2} \mathrm{COONa}\right]$

A plot of diffusion current against concentration again shows a definite break in the line at $0.15 \mathrm{M}$ concentration and $0.172 \mathrm{amp} / \mathrm{cm}^{2}$.

The curves obtained with $0.1 \mathrm{M}$ sodium acetylglycinate known not to give a Kolbe dimer and 0.1 $M$ sodium methoxide in methanol were essentially the same as obtained with sodium acetate in methanol. However, the half-wave potentials, $+2.35 \mathrm{~V}$ and $+2.20 \mathrm{~V}$ vs. silver-silver chloride reference electrode, respectively, were higher than potentials obtained with acids known to give Kolbe products.

\section{Discussion}

As far as can be determined from our experimental results, there is apparently no correlation between structure and half-wave potentials in those acids which yield a Kolbe dimer. If these potentials represent, which we believe they do, the formation of the primary free radical from the ion, then this lack of correlation is not unexpected. Studies on a considerable number of aromatic aldehydes and ketones have shown that there is no correlation between structure and the potential required to induce the formation of the intermediate free radical type required for pinacol formation. However, it is possible to extract one correlation from the experimental date insofar as potential is concerned, and that is it appears Kolbe acids give a lower potential than non-Kolbe acids. If we examine the curves, it can be seen that potassium acetate and sodium iso-valerate produce what might be termed normal polarographic-type curves. By this is meant a clearly defined polarographic step with a flat or gently sloping diffusion current region. At a potential too low for another ion or the solvent to decompose, a flat diffu- 
sion current appears. However, if the half-wave potential of the reacting ion is close to the half-wave potential of another ion or is close to the decomposition potential of the solvent, a sloping diffusion current region usually occurs before the next reaction takes over. Since the half-wave potentials of these acids are close to the decomposition potential of the solvent which is around 2.0 volts, the sloping type diffusion current curve results. Most of the other anions show this type diffusion current curve at low concentration.

However, at higher concentrations these other acids develop a dip in the curve soon after the diffusion current has been reached. Caproate and heptanoate produce these dips even at relatively low concentrations. One possible explanation for this phenomenon is that the non-polar reaction products accumulate at the anode and form an insulating layer which causes an increase in cell resistance, and consequently an increased IR drop. This results in a drop in anode potential with consequent decrease in the rate of anion discharge. Further support for the idea of an insulating product layer is the fact that when the Kolbe reaction is run in an aqueous medium at an anode potential at which water would be expected to decompose, oxygen evolution does not take place preferentially ${ }^{12)}$. This may well be because the aqueous solvent is no longer in contact with the anode, having been replaced at the anode surface by a non-polar product. In addition, an examination of the current-voltage curves where the dip occurs at higher concentrations, e.g., valerate, caproate, and iso-caproate, will show that the rise in current at the higher potentials, which has been attributed to decomposition of methanol, is absent when the dip appears. At lower concentrations the methanol rise is present. This appears to be a good indication that the solvent is no longer in contact with the electrode.

The question now arises as to why this dip does not occur with acetate and iso-valerate ions. To answer this question we must first see what causes product to accumulate at the electrode. Two opposing rates control this phenomenon. The first is the rate at which product is formed. This is controlled by the rate of electrode reaction, and is measured by the current being passed. The second is the rate at which the product diffuses out of the diffusion layer and into the body of the solution. The thickness of the diffusion layer is limited by the stirring rate of the anode.

When the rate of diffusion of product out of the diffusion layer is faster than its rate of formation, no accumulation of product in the diffusion layer occurs. But when the rate of product formation is faster than the rate of diffusion of this product, insulating layer builds up. If the difference in the rates is large, the product layer builds up rapidly and the diffusion current appears as a peak in the curve. When the difference in the two rates is small, the product layer takes time to accumulate, and the diffusion current appears as a gradual curve into the dip, and the dip is not as severe.

The diffusion rate of the product is determined by its diffusion coefficient. From the Stokes-Einstein Equation we know that the diffusion coefficient of an uncharged molecule is inversely proportional to the size of this molecule. The reaction products from the acetate ion and the iso-valerate ion, ethane and 1.5-dimethylhexane, respectively, are relatively small or compact molecules, and therefore have a high diffusion coefficient. With a high diffusion coefficient, the rate at which product diffuses away from the electrode is faster than the rate at which it is formed, and no product layer builds up.

In the cases where a product layer does form, the size of the product molecule is quite large. It seems entirely reasonable that the rate at which a large uncharged molecule diffuses through a polar solvent could be slow enough to interfere with the reaction when one considers the fact that in polarography the diffusion current is being determined by the rate at which a charged particle diffuses through a polar solvent to an oppositely charged electrode.

Examination of the plots of current density vs. concentration showed that sodium acetate gave a straight line, which is what one would anticipate when doing a usual polarographic study. However, obtaining two straight lines is not usual. The factor which controls the slope of the line produced in this type of plot is the diffusion coefficient of the depolarizer. The greater the diffusion coefficient, the 
greater will be the slope of the line produced. Only a change in diffusion coefficient from one value to another could have produced the results above.

In addition to temperature, which is constant in all of these experiments, the factors which control the diffusion coefficient of an ion are viscosity and the polarity of the solvent. Since these are constants for each solvent, only a change in solvents can produce the change in diffusion coefficients observed in these experiments. The methanol which makes up the bulk of the solution could not change, but the nature of the solvent at the electrode surface could change if product diffuses away from the electrode at a rate slower than that at which it is formed. Further evidence in favor of a product layer has been reported by Conway ${ }^{13}$ ) who obtained galvanostatic charging and open circuit decay curves for the Kolbe type reaction. This results indicated the formation of absorded intermediates during the anodic reactions. An insulating product layer would produce the same type of curves.

Assuming that a product layer does form, and that this changes the diffusion coefficient of the depolarizer, the question now arises as to why the change in slopes of the plotted lines is so abrupt. It is here that the concept of critical current density must be mentioned. Although there is some disagreement on this matter ${ }^{14}$, a number of investigators ${ }^{15}$ ) have suggested essentially the same idea. The Kolbe product forms only if a high current density is maintained, and for each acid there is a critical current density below which no Kolbe product will form. Above this current density, a high yield of Kolbe product may often be obtained.

Since current is a measure of rate of reaction, current density will be a measure of the rate of radical formation at the anode. The radical either dimerizes or forms the by-product, depending on its concentration $^{16)}$. Therefore, the current density controls the nature of the product formed. The sharp change in slope from one straight line to the other in the diffusion current-concentration plots occurs at the critical current density. The molecules formed below the critical current density are only half as large as the Kolbe products which form above it. Since molecular size controls the diffusion coefficient of an uncharged molecule, the by-products diffuse rapidly away from the anode, and do not generally form the insulating layer. This explains why the dip in the curve does not usually appear at the lower concentrations where the current density is below the critical point. The larger Kolbe product molecules do not diffuse as rapidly away from the anode, and so they accumulate into a hydrocarbon layer, and the dip usually appears. It should be made clear that the appearance of a dip in the curve is not necessarily an indication of whether or not the Kolbe product is forming. The dip does not appear in the curves for potassium acetate, although the current density is high enough for the Kolbe product to form. As stated before, this is because the product is a small molecule and has a high diffusion coefficient. By the same token, the dip does appear in the lower concentrations of sodium heptanoate, where the current density has not reached the critical point. This is because the by-product is a larger molecule than the by products of some of the other acids. The dip in the curve is only an indication that hydrocarbon is accumulating at the anode.

In the case of sodium heptanoate, both product and by-product accumulate at the anode. The change in slope of the diffusion current $v s$. concentration curve still occurs for the same reasons given previously. In this case, the product layer changes character at the critical current density from one hydrocarbon to another, which has twice the molecular weight of the first. The Kolbe product from heptanoate, dodecane, is so large a molecule that the diffusion coefficient of the depolarizer is very small, and large changes in concentration are needed to produce a measurable change in diffusion current. For this reason, the slope of the line above the critical current density appears to be zero.

In the case of sodium iso-valerate, the branching in the Kolbe product produces a compact molecule which can diffuse away from the anode rapidly, and no product layer forms, as evidenced by the lack of a dip in any of the curves. The slight change observed in the slope of the plot of diffusion current vs. 
concentration with isovalerate required no serious considration. In addition, this is one of the few acids where both product and by-product form in equal amounts at higher current densities ${ }^{17)}$. The theory of a critical current density does not hold here, and no explanation for this has been found. One possibility is a very short radical life, due to the ability of a branched radical to disproportionate so easily, but not enough is known about the factors which control radical disproportionation ${ }^{18)}$. Alpha substituted acids form no Kolbe product ${ }^{14)}$, while sodium iso-caproate, where branching is further removed from. the point of radical formation, behaves normally.

Kolbe product forms only at high concentrations of depolarizers ${ }^{14)}$. The explanation for this fact ties in nicely with the critical current density theory. At low concentrations the normal diffusion current prevents the current density from reaching the critical point. As concentration is increased, diffusion current increases accordingly until finally at a high enough concentration the diffusion current reaches the point of critical current density, and Kolbe product begins to form. One might therefore say that perhaps there exists for each acid a critical concentration below which no Kolbe product will form. However, this does not take into account the area of the anode. It is known that the Kolbe reaction goes poorly at a platinized platinum electrode. The more highly polished the electrode becomes, the more efficient the Kolbe reaction becomes. This may be because a rough surface has a much greater area than a smooth surface has a much greater area than a smooth surface of the same size. Since current density is equal to. the current passed divided by the area of the electrode, a smooth electrode has a much smaller effective area, and therefore the current density is greater.

\section{Conclusion}

In conclusion we might say that based on our experimental results, Kolbe acids give a relatively lower half-wave potential than a non-Kolbe acid, and that this potential may be considered as that potential at which the carboxylate radical is formed. Further, there appears to be a critical current density and concentrationf or optimum formation of dimer from many Kolbe acids.

(Received Feb. 7, 1962).

\section{Literature :}

1) Brown, Walker, Ann. 261, 107 (1891).

2) Fichter, Trans. Amer. Electrochem. Soc. 45, 131 (1924).

3) Glasstone, Hickling, J. Chem. Soc. 1878 (1934).

4) Saluaze, Compt. rend. 180, 662 (1925).

5) Kolbe, Ann. 69, 257 (1849).

6) Glasstone, Hickling, Trans. Amer. Electrochem. Soc. 75, 333 (1939).

7) Wilson, Lippincott, J.A.C.S., 78, 4290 (1956).

8) Brown. J.A.C.S. 56, 646 (1934).

9) Gillet, J. Electrochem. Soc. 108, 71 (1961).

10) M.J. Allen, Canad. J. Chem. 37, 257 (1959).

11) Parson, "Handbook of Electrochemical Constants" p. 74 (1959).

12) M.J. Allen, "Organic Electrode Processes" p. 99 (1958), Reinhold Pub. Corp., New York.

13) Conway, Dzieciuch, "Kinetics of Anodic Processes in Carboxylic Acids" presented before the Division of Theoretical Electrochemistry at the 117th Meeting of Electrochem. Soc., Chicago, I 11., May, 1960.

14) M.J. Allen, "Organic Electrode Process" Chap. VI (1958), Reinhold Pub. Corp., New York.

15) Shukla, Walker, Trans. Faraday Soc. 27, 35 (1934); Ruzicka, Stoll, Helv. Chim. Acta., 17, 1308 (1934); Wilson, Lippincott, "Kolbe Electrosynthesis: Effect of Alternating Current", presented before the Electro-Organic Division at the 108th Meeting of Electrochem. Soc., Pittsburgh, Pa., October, 1955.

16) M.J. Allen, "Organic Electrode Processes" p. 102 (1958), Reinhold Pub. Corp., New York

17) Ibid., p. 103.

18) Walling, "Free Radicals in Solution" p. 33 (1957), John Wiley and Sons., Inc., New York. 